INDO GLOBAL JOURNAL OF

PHARMACEUTICAL SCIENCES

ISSN 2249- 1023

\title{
Approaches to Explore Next Generation Sequencing Data: Focus on microRNAs
}

\author{
Yashika Rustagi *, Vibha Rani \\ Department of Biotechnology, Jaypee Institute of Information Technology, A-10, Sector 62, Noida, Uttar Pradesh-201301, India \\ Address for Correspondance: Yashika Rustagi, yashikarustagi@gmail.com ; vibha.rani@jiiit.ac.in
}

Keywords Next

Generation

Sequencing; Small

RNAs; microRNAs; Targets;

Computational

Methods.

\begin{abstract}
Next generation sequencing has come up with new era of nucleic acid sequencing studies to the lead of current biological data research. High throughput sequencing combined with advance molecular and computational techniques has given researchers to profile the whole genomes sequence of DNA and RNA much more quickly than the previously used Sanger sequencing and pyrosequencing, which have revolutionised the study of genomics and molecular biology. NGS is done by using different modern sequencing technologies/platforms such as Illumina (Solexa) sequencing Roche 454 sequencing Ion torrent: Proton / PGM sequencing, SOLiD sequencing etc. In this technical discussion we aim to explain the strategies and methodologies with briefing the workflow and methods of library preparation, sequencing and analysis techniques that have been developed to profile a specific class of small RNAs i.e. microRNAs using next generation sequencing platforms. MicroRNAs are the class of non-coding small RNAs which regulates gene expression at posttranscriptional level by mRNA degradation or inhibition and act as a potential tiny regulators. We will also describe methodologies of target prediction, GO and KEGG analysis using NGS data. (C) 2016 iGlobal Research and Publishing Foundation. All rights reserved.
\end{abstract}

Conference Proceedings: International Conference on Advances in Plant and Microbial Biotechnology (PMB2017); JIIT, Noida: February 02-04, 2017

Indo Global Journal of Pharmaceutical Sciences( ISSN 22491023 ; CODEN- IGJPAI; NLM ID: 101610675) indexed and abstracted in EMBASE(Elsevier), SCIRUS(Elsevier),CABI, CAB Abstracts, Chemical Abstract Services(CAS), American Chemical Society(ACS), Index Copernicus, EBSCO, DOAJ, Google Scholar and many more. For further details, visit http://iglobaljournal.com 